\title{
Senescence of Rice Leaves XXIV. Involvement of Calcium and Calmodulin in the Regulation of Senescence
}

\author{
Yuanman Huang, Chien Teh Chen and Ching Huei Kao
}

Department of Agronomy, National Taiwan University, Taipei, Taiwan, Republic of China

\begin{abstract}
Effects of compounds that influenced calcium uptake and calmodulin inhibitors on the senescence of detached rice leaves were examined. Chelators, ethyleneglycol-bis-( $\beta$-aminoethyl ether)$N, N, N, N$-tetraacetic acid (EGTA) and 1,2-bis-(o-aminophenoxy)-ethane- $N, N, N, N$-tetraacetic acid (BAPTA), significantly promoted senescence of detached rice leaves in the dark and light. The effect of EGTA can be reversed by treating detached rice leaves with calcium. Verapamil, a calcium channel blocker, and lanthanum chloride, a calcium antagonist, promoted dark-induced, and suppressed BA- and light-retarded senescence of detached rice leaves. Calcium ionophore A23187 and ruthenium red, believed to raise cytosolic level of $\mathrm{Ca}^{2+}$, were quite effective in retarding dark-induced and ABA-promoted senescence of detached rice leaves. Calmodulin inhibitors, W-7, compound 48/80, chlorpromazine and trifluoperazine, significantly promoted dark-induced, and suppressed BA- and light-retarded senescence of detached rice leaves. It is concluded that cytosolic level of $\mathrm{Ca}^{2+}$ may regulate senescence of detached rice leaves through a calmodulindependent mechanism.
\end{abstract}

Key words: Calcium - Calmodulin - Leaf senescence - Oryza sativa.

Calcium is known to retard ripening process of fruit. Specifically, maintenance of relatively high calcium concentrations in fruit tissue results in a slower rate of ripening, as seen in lower respiration, reduced ethylene production, and slower softening of fruit flesh (Ferguson 1984). Leaf senescence has also been shown to be retarded by the application of calcium. Chl loss and protein degradation were both reduced in corn and Rumex leaf discs by calcium (Poovaiah and Leopold 1973). In whole, excised cucumber cotyledons, calcium treatment reduced the rate of $\mathrm{Chl}$ degradation and peroxide accumulation (Ferguson et al. 1983). They related the calcium effect to maintain cellular membranes (Ferguson et al. 1983, Poovaiah and Leopold 1973). It appears that the senescence-effect of calcium is an effect of external calcium. Using pea leaf system, Leshem et al. $(1982,1984,1986)$ were able to show that senescence was promoted by increasing intracellular calcium. They concluded that elevated intracellular calcium promoted senescence through a calmodulin-mediated effect.

Abbreviations: BA, benzyladenine; BAPTA, 1,2-bis-(oaminophenoxy)-ethane- $N, N, N, N$-tetraacetic acid; $\mathrm{CPZ}$, chlorpromazine; EGTA, ethyleneglycol-bis-( $\beta$-aminoethyl ether)$N, N, N, N$-tetraacetic acid; TFPZ, trifluoperazine.
The present investigation was undertaken to examine the effects of calcium chloride, calcium chelators (EGTA and BAPTA), a calcium antagonist (lanthanum chloride), a calcium channel blocker (verapamil), a calcium ionophore (A23187), and anti-calmodulin drugs (W-7, compound $48 / 80, C P Z$ and TFPZ) on the senescence of detached rice leaves. Results indicate that elevated cytosolic calcium retards senescence of detached rice leaves through a calmodulin-dependent mechanism.

\section{Materials and Methods}

Rice (Oryza sativa cv. Taichung Native 1) seedlings were cultured as previously described (Kao 1980). The apical 3-cm segments excised from the third leaves of 12 day-old seedlings were used. A group of 10 segments were floated in a Petri dish containing $10 \mathrm{ml}$ of test solutions. All chemicals used in this investigation were purchased from Sigma. Incubation was carried out at $27^{\circ} \mathrm{C}$ in darkness or the light $\left(16.7 \mathrm{~W} \cdot \mathrm{m}^{-2}\right)$ provided by fluorescent lamps.

Chl was extracted and quantitated as described previously (Kao 1980). All experiments were repeated at least twice. Data are presented as the results of a single experi- 
ment typical of the trends seen in the repeated experiments.

\section{Results}

The effectiveness of calcium in retarding $\mathrm{Chl}$ degradation of detached rice leaves was tested as shown in Figure 1. Calcium was effective in retarding the decrease of $\mathrm{Chl}$ under both light and dark conditions. The effect of calcium in the dark was more effective than that in the light. The effect of EGTA on Chl content of detached rice leaves in the dark is presented in Figure 2. The amount of Chl was decreased by EGTA treatment. This effect was reversed by treating detached rice leaves with calcium (data not shown). $\mathrm{Ca}^{2+}$ chelator BAPTA was found to be more effective than EGTA in decreasing Chl content (Fig. 2).

Experiments were carried out with putative calcium channel blocker to further characterize the role of calcium in the regulation of senescence of detached rice leaves in the dark. Verapamil, a calcium channel blocker, or lanthanum chloride, a calcium antagonist, was applied to detached rice leaves in the dark. The results in Figure 3 indicate that verapamil was very effective in causing the decrease of $\mathrm{Chl}$ content. Lanthanum chloride also decreased $\mathrm{Chl}$ content in the dark (Fig. 3). These experiments suggest that Chl loss of detached rice leaves in the dark is regulated by a block of transport of calcium ions into the cytosol. Further evidence of this role for calcium was obtained using the calcium ionophore A23187 (Campbell 1983). Treatment with the ionophore at $0.01 \mathrm{~mm}$ resulted in higher Chl content than the control (Table 1). Ruthenium red is known to block calcium ion transport (Hinds et al. 1981, Watson et al. 1971) and is believed to

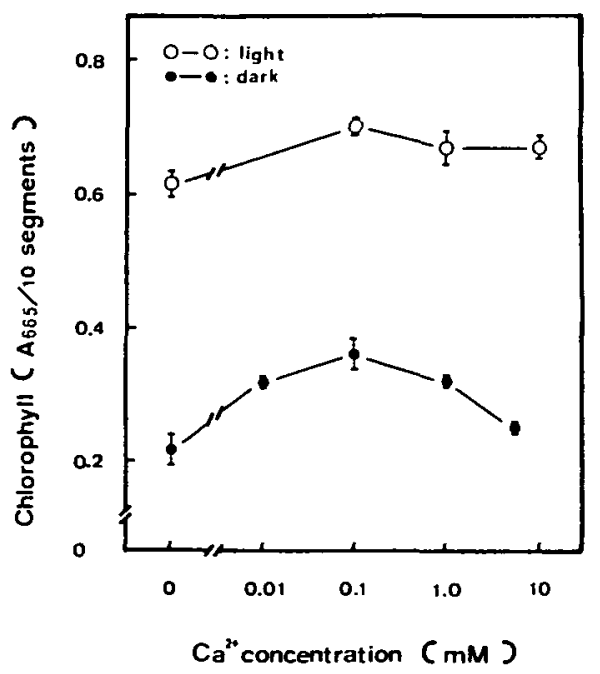

Fig. 1 Effect of calcium chloride on the $\mathrm{Chl}$ content of detached rice leaves. Chl was determined after 4 days in the dark or light. Values are averages with standard errors $(n=4)$.

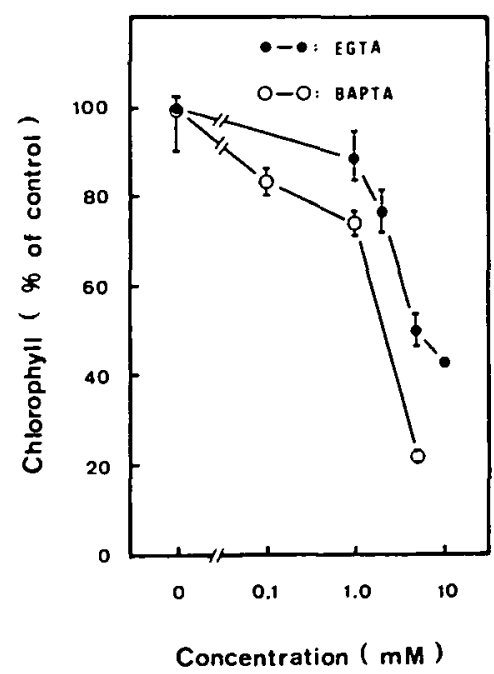

Fig. 2 Effects of EGTA and BAPTA on the Chl content of detached rice leaves in the dark. Chl was determined after 4 days in the dark. Values are averages with standard errors $(n=4)$.

raise the intracellular calcium level (Bednarska 1989, Moll and Jones 1982, Vashington et al. 1972). We investigated the effect of this compound on the Chl content of detached rice leaves in the dark. Ruthenium red was found very effective in retarding $\mathrm{Chl}$ loss (Table 1).

We also investigated the Chl content of detached rice leaves in the dark in the presence of calmodulin antagonists. Results shown in Table 1 and Figure 4 indicate that calmodulin antagonists, W-7, compound $48 / 80, \mathrm{CPZ}$ and TFPZ, significantly accelerated the decrease of Chl con-

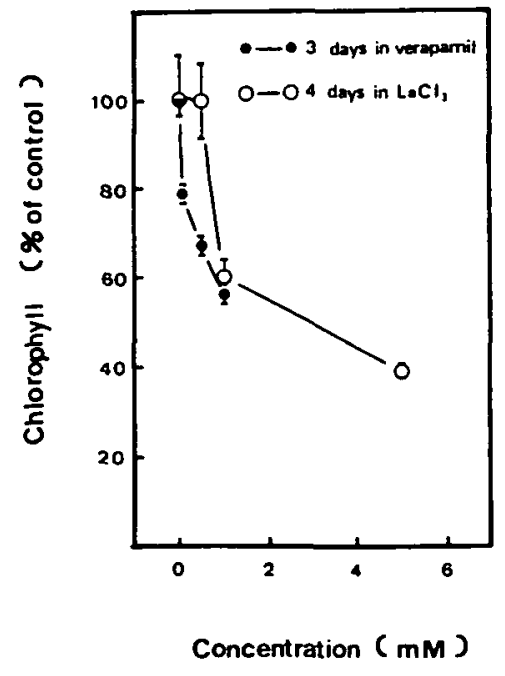

Fig. 3 Effects of verapamil and lanthanum chloride on the Chl content of detached rice leaves in the dark. Values are averages with standard errors $(n=4)$. 
Table 1 Effect of A23187, ruthenium red, W-7 and compound $48 / 80$ on the $\mathrm{Chl}$ content of detached rice leaves in the dark

\begin{tabular}{cc}
\hline Treatment & Chl $\left(A_{665} / 10\right.$ segments $)$ \\
\hline A23187 (mM) & $0.44 \pm 0.01$ \\
0 & $0.67 \pm 0.02$ \\
0.01 & \\
Ruthenium red (mM) & $0.40 \pm 0.02$ \\
0 & $0.49 \pm 0.04$ \\
0.5 & $0.61 \pm 0.02$ \\
1.0 & $0.71 \pm 0.03$ \\
2.0 & \\
W-7 (mM) & $0.72 \pm 0.03$ \\
0 & $0.46 \pm 0.02$ \\
0.1 & $0.34 \pm 0.03$ \\
0.5 & \\
Compound $48 / 80(\mu \mathrm{g} / \mathrm{ml})$ & $0.55 \pm 0.03$ \\
0 & $0.43 \pm 0.01$ \\
\hline 00 & \\
\hline
\end{tabular}

Chl was determined after 4 days in the dark. Values are averages with standard errors $(n=4)$.

tent.

$\mathrm{BA}$, a synthetic cytokinin, is known to retard $\mathrm{Chl}$ degradation of detached rice leaves in the dark (Kao 1980). To see whether there is a requirement for calcium channels when BA retards Chl degradation, agents known to block such channels were incorporated in the incubation media. Lanthanum chloride and verapamil significantly reduced the retardation effect of $\mathrm{Chl}$ loss by BA (Table 2). We also

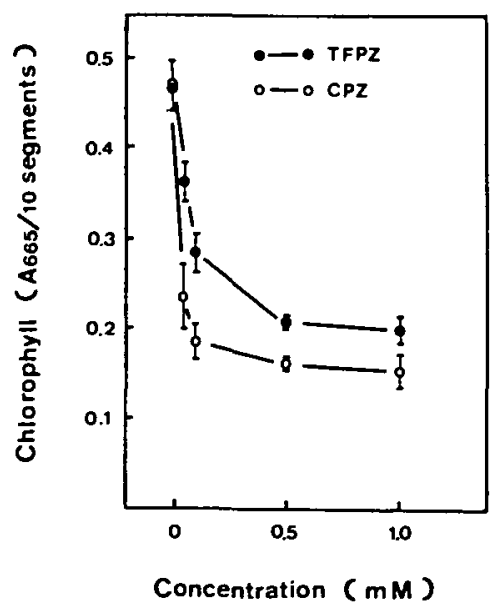

Fig. 4 Effects of TFPZ and CPZ on the Chl content of detached rice leaves in the dark. Chl was determined after 4 days in the dark. Values are averages with standard errors $(n=4)$.
Table 2 Effects of BA alone and BA in the presence lanthanum chloride, verapamil, CPZ, TFPZ, W-7 and compound $48 / 80$ on the $\mathrm{Chl}$ content of detached rice leaves in the dark

\begin{tabular}{lc}
\hline Treatment & Chl $\left(A_{665} / 10\right.$ segments $)$ \\
\hline Control & $0.23 \pm 0.02$ \\
BA & $0.78 \pm 0.03$ \\
BA + $\mathrm{LaCl}_{3}$ & $0.55 \pm 0.02$ \\
BA+Verapamil & $0.29 \pm 0.03$ \\
BA+CPZ & $0.45 \pm 0.02$ \\
BA+TFPZ & $0.39 \pm 0.02$ \\
Control & $0.72 \pm 0.03$ \\
BA & $0.95 \pm 0.02$ \\
BA+W-7 & $0.84 \pm 0.03$ \\
Control & $0.55 \pm 0.03$ \\
BA & $0.96 \pm 0.01$ \\
BA + Compound $48 / 80$ & $0.82 \pm 0.01$ \\
\hline
\end{tabular}

The concentrations for BA, lanthanum chloride, verapamil, and CPZ are $1 \mathrm{~mm}$, whereas those for TFPZ, W-7 and compound 48/ 80 are $0.5 \mathrm{~mm}, 0.1 \mathrm{~mm}$ and $100 \mu \mathrm{g} / \mathrm{ml}$, respectively. Chl was determined after 4 days in the dark. Values are averages with standard errors $(n=4)$.

investigated the ability of BA to retard Chl loss in the presence of calmodulin antagonists in the dark. In the presence of W-7, compound 48/80, CPZ and TFPZ, the effect of BA was significantly suppressed (Table 2 ), indicating there is a requirement of calmodulin for BA to retard $\mathrm{Chl}$ loss of detached rice leaves in the dark.

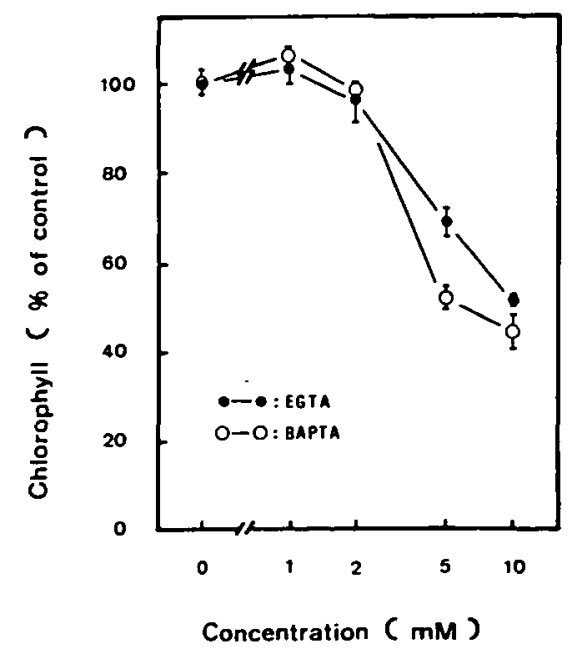

Fig. 5 Effects of EGTA and BAPTA on the Chl content of detached rice leaves in the light. $\mathrm{Chl}$ was determined after 4 days in the light. Values are averages with standard errors $(n=4)$. 
Table 3 Effects of lanthanum chloride, verapamil, CPZ, TFPZ, W-7 and compound $48 / 80$ on the Chl content of detached rice leaves in the light

\begin{tabular}{lc}
\hline Treatment & Chl $\left(A_{665} / 10\right.$ segments $)$ \\
\hline Control & $0.56 \pm 0.02$ \\
LaCl $_{3}$ & $0.34 \pm 0.02$ \\
Verapamil & $0.21 \pm 0.12$ \\
CPZ & $0.46 \pm 0.01$ \\
TFPZ & $0.45 \pm 0.01$ \\
Control & $0.80 \pm 0.01$ \\
W-7 & $0.59 \pm 0.01$ \\
Control & $0.79 \pm 0.01$ \\
Compound $48 / 80$ & $0.64 \pm 0.02$ \\
\hline
\end{tabular}

The concentrations for lanthanum chloride, verapamil and $\mathrm{CPZ}$ are $1 \mathrm{mM}$, wherease those for TFPZ, W-7 and compound 48/80 are $0.5 \mathrm{~mm}, 0.1 \mathrm{mM}$ and $100 \mu \mathrm{g} / \mathrm{ml}$, respectively. Chl was determined after 4 days in the light. Values are averages with standard errors $(n=4)$.

Light has been shown to retard Chl loss of detached rice leaves (Hurng et al. 1986). Similar to the effect of BA in the dark, calcium and calmodulin appear to be required for the effect of light. This conclusion is supported by the observations that calcium chelators, calcium channel blockers and calmodulin antagonists significantly reduced the effect of light (Fig. 5 and Table 3).

ABA significantly promoted $\mathrm{Chl}$ loss of detached rice leaves in the light (Table 4). ABA effect was totally abolished by ruthenium red and significantly reduced by A23187 (Table 4). These experiments seem to suggest that ABA promoted Chl loss may mediate through blocking calcium ions into the cytosol.

Table 4 Effects of $\mathrm{ABA}$ alone and $\mathrm{ABA}$ in the presence of ruthenium red or $\mathrm{A} 23187$ on the Chl content of detached rice leaves in the light

\begin{tabular}{lc}
\hline Treatment & $\mathrm{Chl}\left(A_{665} / 10\right.$ segments $)$ \\
\hline Control & $0.88 \pm 0.01$ \\
ABA & $0.26 \pm 0.02$ \\
ABA + Ruthenium red & $0.87 \pm 0.03$ \\
Control & $0.58 \pm 0.03$ \\
ABA & $0.36 \pm 0.01$ \\
ABA + A23187 & $0.43 \pm 0.02$
\end{tabular}

The concentrations of ABA, ruthenium red and A23187 are 0.1, 2.0 and $0.01 \mathrm{~ms}$, respectively. Chl was determined after 4 days in the light. Values are averages with standard errors $(n=4)$.

\section{Discussion}

Several compounds expected to increase or decrease cytosolic levels of calcium were used to examine the requirement of calcium in regulating the senescence of detached rice leaves, measured as $\mathrm{Chl}$ loss. We also investigated effects of four inhibitors known to inhibit action of $\mathrm{Ca}$ calmodulin in animals.

EGTA is a specific $\mathrm{Ca}^{2+}$ chelator (Campbell 1983) used in many systems to affect endogenous $\mathrm{Ca}^{2+}$ concentrations (Daye 1984, Karege et al. 1982, Lehtonen 1984) by causing a depletion of cytosolic $\mathrm{Ca}^{2+}$ (Campbell 1983, Gilroy et al. 1986, Poovaiah and Reedy 1987). The chelator BAPTA is much more selective for $\mathrm{Ca}^{2+}$ than EGTA (Tsien 1980). In the present study, we found that both EGTA and BAPTA were effective in promoting $\mathrm{Chl}$ loss. The effect of EGTA could be reversed by the addition of Ca.

Of particular interest is the finding that ruthenium red, a hexavalent dye, significantly retarded dark-induced and ABA-promoted senescence of detached rice leaves. Ruthenium red has been shown to be a potent inhibitor of $\mathrm{Ca}^{2+}$ uptake and efflux by animal cells and mitochondria (Hinds et al. 1981, Watson et al. 1971). This compound also intereferes with $\mathrm{Ca}^{2+}$ efflux from aleurone layers of barley (Moll and Jones 1983). Recently, Bednarska (1989) reported that ruthenium red raised the amount of ${ }^{45} \mathrm{Ca}^{2+}$ taken up by germinating pollen grains relative to the control. Based on the known mechanism of ruthenium red action in animal and plant cells, the most likely explanation of its effect on Chl loss involves its role in the regulation of cytosolic $\mathrm{Ca}^{2+}$ content.

The importance of the cytosolic $\mathrm{Ca}^{2+}$ in the regulation of rice leaf senescence is further supported by the observations that (a) lanthanum chloride, a calcium antagonist, and verapamil, a calcium channel blocker, promoted darkinduced, and reduced light- and BA-retarded senescence of detached rice leaves, and (b) calcium ionophore A23187, a compound that induces an increases in the cytosolic $\mathrm{Ca}^{2+}$, retarded dark-induced and ABA-promoted senescence of detached rice leaves.

Our data thus strongly suggest that an elevated level of cytosolic level of $\mathrm{Ca}^{2+}$ is required for retarding senescence of detached rice leaves. However, our results are inconsistent with those reported by Leshem et al. (1982, 1984, 1986), who found that senescence of pea leaves was promoted by increasing cytosolic $\mathrm{Ca}^{2+}$. The use of different plant species may have led this discrepancy. Recently, Leshem (1987) proposed that elevated cytosolic $\mathrm{Ca}^{2+}$ might promote senescence through a mechanism that triggered the catabolic process by binding to calmodulin and activating phospholipase $\mathbf{A}_{2}$.

The use of calmodulin inhibitors on intact cells can lead to complex responses, and it cannot be assumed that 
the primary target reaction or system is the only one affected. It was for this reason that we used four different calmodulin inhibitors, and it is important that we obtained essentially similar results with all four. The concentrations of calmodulin inhibitors used in this investigation are comparable with those used by others who concluded that Ca-calmodulin is probably essential for physiological processes (Elliott 1983, Elliott et al. 1983, Friedman et al. 1989, Muto and Hirosawa 1987). Furthermore, no toxicity (rolling or loss of turgor) was visually observable at the inhibitory concentrations.

Since calmodulin inhibitors were found to promote dark-induced and reduce $\mathrm{BA}$ - and light-retarded senescence, the cytosolic $\mathrm{Ca}^{2+}$ seems to regulate senescence through a calmodulin-mediated effect. Calcium- and calmodulin-stimulated phosphorylation of membrane proteins has been found to decrease during senescence of apples (Paliyath and Poovaiah 1984, 1985). In a recent study, we demonstrated that proton secretion activity of detached rice leaves, which was found to be originated from ATP-driven $\mathrm{H}^{+}$pump located in the plasma membrane, played an important role in regulating senescence of detached rice leaves (Chen et al. 1990). Proton secretion activity decreased during senescence of detached rice leaves. Phosphorylation of the plasma-membrane $\mathrm{H}^{+}$-ATPase has been shown to be regulated by calcium-stimulated protein kinase (Bidwai and Takemoto 1987, Schaller and Sussman 1988). Hanson and Trewavas (1982) suggested that calcium and calmodulin might regulate the activity of $\mathrm{H}^{+}$-ATPase of the plasma membrane. It seems that the promotion of $\mathrm{Ca}^{2+}$ and calmodulin-dependent protein kinase or phosphorylation by $\mathrm{Ca}^{2+}$ and calmodulin provides a mechanism by which intracellular $\mathrm{Ca}^{2+}$ can regulate senescence of detached rice leaves. The possible changes of protein kinase activity or protein phosphorylation during senescence of detached rice leaves are now the subject of further research.

This research was supported financially by the National Science Council, Republic of China under Grant NSC 79-0409B002-04.

\section{References}

Bednarska, E. (1989) The effect of exogenous $\mathrm{Ca}$ ions on pollen grain germination and pollen growth. Investigations with ${ }^{45} \mathrm{Ca}^{2+}$ together with verapamil, $\mathrm{La}^{3+}$, and ruthenium red. Sexual Plant Reproduction 2: 53-58.

Bidwai, A. P. and Takemoto, J. Y. (1987) Bacterial phytotoxin, syringomycin, stimulates a protein kinase mediated phosphorylation of red beet plasma membrane polypeptides (Abstr.). J. Cell Biochem. (Suppl.) 11B: 93.

Campbell, A. K. (1983) Intracellular Calcium: Its Role as
Regulator. p. 537, Wiley, New York.

Chen, C. T., Chou, I. T. and Kao, C. H. (1990) Senescence of rice leaves $\mathrm{XX}$. Changes of proton secretion during senescence. Plant Sci. 66: 29-34.

Daye, S., Bico, R. L. and Roux, S. J. (1984) Inhibition of gravitropism in oat coleoptiles by the calcium chelator, ethyleneglycol-bis-( $\beta$-aminoethylether)- $N, N$-tetraacetic acid. Physiol. Plant. 61: 449-454.

Elliott, D. C. (1983) Inhibition of cytokinin-regulated responses by calmodulin-binding compounds. Plant Physiol. 72: 215218.

Elliott, D. C., Batchelor, S. M., Cassar, R. A. and Marinos, N. G. (1983) Calmodulin-binding drugs affect responses to cytokinin, auxin, and gibberellic acid. Plant Physiol. 72: 219-224.

Ferguson, I. B. (1984) Calcium in plant senescence and fruit ripening. Plant Cell Environ. 7: 477-489.

Ferguson, I. B., Watkins, C. B. and Harman, J. E. (1983) Inhibition by calcium of senescence of detached cucumber cotyledons: effect on ethylene and hydroperoxide production. Plant Physiol. 71: 182-186.

Friedman, H., Goldschmidt, E. E. and Halevy, A. H. (1989) Involvement of calcium in the photoperiodic flower induction process of Pharbitis nil. Plant Physiol. 89: 530-534.

Gilory, S., Hughes, W. A. and Trewavas, A. J. (1986) The measurement of intracellular calcium level in protoplasts from higher plant cell. FEBS Lett. 199: 217-223.

Hanson, J. B. and Trewavas, A. J. (1982) Regulation of plant cell growth: The changing perspective. New Phytol. 90: 1-18.

Hinds, T. R., Raess, B. U. and Vincenzi, F. F. (1981) Plasma membrane $\mathrm{Ca}^{2+}$ transport: antagonism by several potential inhibitors. J. Membr. Biol. 58: 57-65.

Hurng, W. P., Su, L. Y. and Kao, C. H. (1986) Senescence of rice leaves. XVI. Regulation by light. Bot. Bull. Academia Sinica 27: 163-174.

Kao, C. H. (1980) Senescence of rice leaves. IV. Influence of benzyladenine on chlorophyll degradation. Plant Cell Physiol. 21 : 1255-1262.

Karage, F., Pencel, C. and Greppin, H. (1982) Rapid correlation between the leaves of spinach and the photocontrol of peroxidase activity. Plant Physiol. 69: 437-441.

Lehtonen, J. (1984) The significance of $\mathrm{Ca}^{2+}$ in the morphogenesis of Micrasterias studied with EGTA, verapamil, $\mathrm{LaCl}_{3}$ and calcium ionophore A23187. Plant Sci. Lett. 33: 5360 .

Leshem, Y. Y. (1987) Membrane phospholipid catabolism and $\mathrm{Ca}^{2+}$ activity in control of senescence. Physiol. Plant. 69: 551559 .

Leshem, Y. Y., Wurzburger, Y., Frimer, A. A., Barness, G. and Ferguson, I. B. (1982) Calcium and calmodulin metabolism in senescence: interaction of lipoxygenase and superoxide dismutase with ethylene and cytokinin. In Plant Growth Substances 1982. Edited by Wareing, P. F. pp. 569-578. Academic Press, London.

Leshem, Y. Y., Sridhara, S. and Thompson, J. E. (1984) Involvement of calcium and calmodulin in membrane deterioation dur- 
ing senescence of pea foliage. Plant Physiol. 75: 329-335.

Leshem, Y. Y., Frend-Silverberg, M., Warzburger, J., Bar-Nes, G., Malik, Z. and Langsam, Y. (1986) $\mathrm{Ca}^{2+}$ : calmodulin phytohormone-linked plant senescence control. In Plant Growth Substances 1985. Edited by Bopp, M. pp. 159-168. Springer-Verlag, Berlin.

Moll, B. A. and Jones, R. L. (1982) a-Amylase secretion by single barley aleurone layer. Plant Physiol. 70: 1149-1155.

Muto, S. and Hirosawa, T. (1987) Inhibition of adventitious root growth in Tradescantia by calmodulin antagonists and calcium inhibitors. Plant Cell Physiol. 28: 1569-1574.

Paliyath, G. and Poovaiah, B. W. (1984) Calmodulin-inhibitor in senescing apples and its physiological and pharmacological significances. Proc. Natl. Acad. Sci. USA 81: 2065-2069.

Paliyath, G. and Poovaiah, B.W. (1985) Calcium- and calmodulin-promoted protein phosphorylation of membrane proteins during senescence in apples. Plant Cell Physiol. 26: 977-986.
Poovaiah, B. W. and Leopold, A. C. (1973) Deferral of leaf senescence with calcium. Plant Physiol. 52: 235-239.

Poovaiah, B.W. and Reedy, R.W. (1987) Calcium messenger systems in plants. CRC Crit. Rev. Plant Sci. 6: 47-103.

Schaller, G. E. and Susman, M. R. (1988) Phosphorylation of the plasma-membrane $\mathrm{H}^{+}$-ATPase of oat roots by calcium-stimulated protein kinase. Planta 173: 509-519.

Tsien, R. Y. (1980) New calcium indicators and buffers with high selectivity against magnesium and protons: design, synthesis, and properties of prototype structures. Biochemistry. 19: 2396-2404.

Vasington, F. D., Gazzotti, P. and Cavafoli, E. (1972) The effect of rutehium red on $\mathrm{Ca}$ transport and respiration in rat liver mitochondria. Biochim. Biophys. Acta 256: 43-54.

Watson, E. L., Vincenzi, F. F. and Davis, P. W. (1971) $\mathrm{Ca}^{2+}$-activated ATPase: selective inhibition by ruthenium red. Biochim. Biophys. Acta 249: 606-610.

(Received June 13, 1990; Accepted August 3, 1990) 